CERN-TH/99-177

\title{
TWO-PARTICLE CORRELATIONS FROM THE $q$-BOSON VIEWPOINT
}

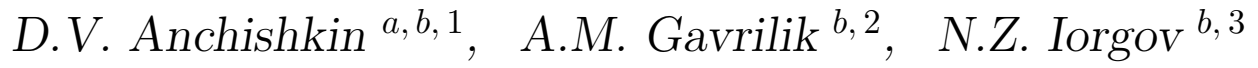 \\ ${ }^{a}$ CERN TH-Division, CH-1211 Geneva 23, Switzerland \\ ${ }^{b}$ Bogolyubov Institute for Theoretical Physics \\ National Academy of Sciences of Ukraine \\ 252143 Kiev-143, Ukraine
}

\begin{abstract}
We propose and develop to some extent a novel approach, which allows us to effectively describe, for relativistic heavy-ion collisions, the empirically observed deviation from unity of the intercept $\lambda$ (i.e. the measured value corresponding to zero relative momentum $\mathbf{p}$ of two registered identical pions or kaons) of the two-particle correlation function $C(p, K)$. The approach is based on the use of two versions of the so-called $q$-deformed oscillators and the corresponding picture of ideal gases of $q$-bosons. By these techniques the intercept $\lambda$ is put into direct correspondence with the deformation parameter $q$. For fixed deformation strength, the model predicts dependence of the intercept $\lambda$ on the pion pair mean momentum $\mathbf{K}$.
\end{abstract}

\section{Introduction}

The hadron matter under intense conditions of high temperatures and densities has been extensively studied with the use of relativistic heavy-ion collisions (RHIC). The insight into the extreme matter with experimentally controlled initial energies serves as an examination of existing models and theories. On the other hand, RHIC promises to be a laboratory where a search for new physics that extends beyond current imaginations can be made.

The models and approaches that are used to describe the processes occurring in the reaction region are examined by comparing provided predictions with experimental data on single-, two-

\footnotetext{
${ }^{1}$ E-mail: Dmitry.Anchishkin@cern.ch or/and Dmitry.Anchishkin@ap3.bitp.kiev.ua

${ }^{2}$ E-mail: omgavr@bitp.kiev.ua

${ }^{3}$ E-mail: mmtpitp@bitp.kiev.ua
} 
and many-particle momentum spectra, which contain information on the source at the early stage (photons, dileptons) and at the stage of so-called "freeze-out" (hadron spectra). Twoparticle correlations encapsulate information about the space-time structure and dynamics of the emitting source [1]-[3]. Usually, consideration of the correlations that occur in relativistic heavy ion-collisions assumes that: (i) the particles are emitted independently (or the source is completely chaotic), and (ii) finite multiplicity corrections can be neglected. Then, correlations reflect a) the effects from symmetrization (antisymmetrization) of the amplitude to detect identical particles with certain momenta, and b) the effects that are generated by the final state interactions of the detected particles between them and with the source. At first sight, the final state interactions (FSI) can be regarded as a contamination of "pure" particle correlations. However, it should be noted that the FSI depend on the structure of the emitting source and thus provide information about source dynamics as well [4]. Discussion of the latter is beyond the scope of the present paper.

The nominal quantity expressing the correlation function in terms of experimental distributions [2] is

$$
C\left(\mathbf{k}_{a}, \mathbf{k}_{b}\right)=\frac{P_{2}\left(\mathbf{k}_{a}, \mathbf{k}_{b}\right)}{P_{1}\left(\mathbf{k}_{a}\right) P_{1}\left(\mathbf{k}_{b}\right)},
$$

where $P_{1}(\mathbf{k})=E d^{3} N / d^{3} k$ and $P_{2}\left(\mathbf{k}_{a}, \mathbf{k}_{b}\right)=E_{a} E_{b} d^{6} N /\left(d^{3} k_{a} d^{3} k_{b}\right)$ are single- and two-particle cross-sections, $\mathbf{k}_{a}$ and $\mathbf{k}_{b}$ being on-shell asymptotic momenta.

In the absence of FSI, for a chaotic source, the correlation function can be expressed as (see Appendix):

$$
C(p, K)=1+\cos \alpha \frac{\left|\int d^{4} X e^{i p \cdot X} S(X, K)\right|^{2}}{\int d^{4} X S\left(X, K+\frac{p}{2}\right) \int d^{4} Y S\left(Y, K-\frac{p}{2}\right)},
$$

where 4-momenta $K$ and $p$ defined as

$$
K=\frac{1}{2}\left(k_{a}+k_{b}\right), \quad p=k_{a}-k_{b} .
$$

The source function $S(x, K)$ (single-particle Wigner density) is defined by emitted singleparticle states $\psi_{\gamma}(t, \mathbf{x})$ at freeze-out times:

$$
S(Y, K)=\int d^{4} y e^{i K \cdot y} \sum_{\gamma, \gamma^{\prime}} \rho_{\gamma \gamma^{\prime}} \psi_{\gamma}\left(Y+\frac{y}{2}\right) \psi_{\gamma^{\prime}}^{*}\left(Y-\frac{y}{2}\right)
$$

where the summation (averaging) is taken over the set of all quantum numbers $\{\gamma\}$ carried by the particle just before it is frozen out. The source freeze-out density matrix $\rho_{\gamma \gamma^{\prime}}$ is the weight factor of such an averaging and depends on the particular model of source, for instance, thermal density operator is widely exploited.

For the system of identical particles that we are going to consider, the two-particle wave function appears to be a symmetrized (antisymmetrized) construction of single-particle states (chaoticity assumption), which reads

$$
\psi_{\gamma_{a} \gamma_{b}}\left(\mathbf{x}_{a}, \mathbf{x}_{b}, t_{a}\right)=\frac{1}{\sqrt{2}}\left[\psi_{\gamma_{a}}\left(\mathbf{x}_{a}, t_{a}\right) \psi_{\gamma_{b}}\left(\mathbf{x}_{b}, t_{a}\right)+e^{i \alpha} \psi_{\gamma_{a}}\left(\mathbf{x}_{b}, t_{a}\right) \psi_{\gamma_{b}}\left(\mathbf{x}_{a}, t_{a}\right)\right]
$$


where $\alpha=0$ for identical bosons, and $\alpha=\pi$ for identical fermions. Note that the function (5) is taken at freeze-out times (about translation from detector times $t \rightarrow \infty$ to emission times, see Appendix).

From now on we shall refer our consideration of two-particle correlations to identical bosons (pions, kaons, etc.). As follows from Eq. (2)), the boson correlation function should approach the exact value two as the relative momentum approaches zero. But as it was observed, from the very first experimental data and up to the most recent experiments, the measured correlation function never reaches this value at $\mathbf{p}=0$. To remove this discrepancy, the correlation function of identical bosons is always taken in the form

$$
C(p, K)=1+\lambda f(p, K)
$$

where $\lambda$ is drawn from an experimental fit to the data, usually in the range $\lambda=0.4-0.9$; $f(p, K)$ is commonly taken as a Gaussian function (in any case, $f(\mathbf{p}=0, K)=1$ ). The deviation of $\lambda$ from unity in RHIC can be explained by the production of secondary pions from resonance decays which are outside the fireball. The presence of long-lived resonances results in an increase of the measured source size and life-times [5, 6].

We are now coming to the key idea of our paper. Confining ourselves to formula (2) to try to explain experimental data, it is then straightforward to put into correspondence the parameter $\lambda$ with the angle $\alpha$, so as to get, by means of $\cos \alpha$, the right reduction factor $\lambda$. Actually, in Eq. (5) one can take the phase factor $e^{-i \alpha}$ in place of the factor $e^{i \alpha}$. However, by simple algebra the two-particle amplitude (52) can be reduced to the form which results in the same two-particle probability as the former one. Indeed, the correlation function (2), which is a measurable quantity, is obviously symmetric with respect to $\alpha \rightarrow-\alpha$. It turns out that an effective symmetrization of a two-particle wave function in heavy-ion collisions exhibits similar features to what one encounters in the description of the Aharonov-Bohm effect, or in the physics of anyons. This means that the two-particle wave function of a boson pair released from a dense and hot environment effectively acquires an additional phase. Hence, the drawn phenomenon can be ascribed to the properties of the medium formed in RHIC, which, as we see, exhibits some non-standard QFT behaviour through the considered correlation functions. So, adopting as a driving idea the fact that the correlation function approaches $1+\lambda$ when the two-boson relative momentum approaches zero, we will attempt to construct an effective model capable to mimic the real physical picture. To perform this, we shall use as our basic tool the so-called (algebra of) q-deformed commutation relations, or techniques of $q$-boson statistics, which certainly can be put in connection with the symmetrization rules.

The deformation parameter $q$ is viewed as an effective (not universal) parameter which efficiently encapsulates most essential features of complicated dynamics of the system under study. In many cases, usage of appropriate $q$-algebra allows one to reduce the treatment of complex system of interacting particles to consideration of a system of non-interacting ones at the price of complication (deformation) of the commutation relations. As an example let us mention the application of $q$-deformed algebras to description of rotational spectra of superdeformed nuclei [7] - here $q$ has different value for each nucleus.

It is worth noting that in the context of hadron theory, q-deformed algebras (or quantum algebras) were also already applied. Such a usage proved to yield a significantly improved 
description of hadron characteristics, both regarding hadron scattering [8]-10] - nonlinearity of Regge trajectories - and in the sector of such static properties as hadron masses and mass sum rules [11, 12].

In what follows, we shall exploit, for the system of pions or kaons, the (ideal) $q$-Bose gas picture based on two concrete versions of $q$-bosons. From the viewpoint of direct physical meaning and/or explanation of the true origin of the $q$-deformation in the considered phenomenon, these versions differ from each other, first of all in the question of whether $q$ must be real or can also take such complex values as a pure phase. Note that, at this stage, we do not go into details concerning the diversity of other, than the above-mentioned, "microscopical" reasons (certainly, not completely unrelated) for the appearance of $q$-deformed statistics. Suffice it to mention that the composite nature of the particles (pseudoscalar mesons) under study may as well result [13, 14] in the $q$-deformed structures linked to the real deformation parameter $q$.

\section{The two versions of q-bosons}

In this section we give a brief sketch of main features of the two versions (type "A" and type "B") of multimode $q$-oscillators, which will be used in subsequent treatment.

\section{$\underline{\text { Type A }}$}

The $q$-oscillators of this type are defined by means of the relations [15]

$$
\begin{gathered}
{\left[a_{i}, a_{j}\right]=\left[a_{i}^{\dagger}, a_{j}^{\dagger}\right]=0, \quad\left[\mathcal{N}_{i}, a_{j}\right]=-\delta_{i j} a_{j}, \quad\left[\mathcal{N}_{i}, a_{j}^{\dagger}\right]=\delta_{i j} a_{j}^{\dagger}, \quad\left[\mathcal{N}_{i}, \mathcal{N}_{j}\right]=0,} \\
a_{i} a_{j}^{\dagger}-q^{\delta_{i j}} a_{j}^{\dagger} a_{i}=\delta_{i j} .
\end{gathered}
$$

Note that, if $i \neq j$, this system of independent $q$-oscillators differs essentially from quons [16] whose different modes are non-commuting (i.e. $q$-commuting).

From the vacuum state given by $a_{i}|0,0, \ldots\rangle=0$ for all $i$, the state vectors

$$
\left|n_{1}, n_{2}, \ldots, n_{i}, \ldots\right\rangle \equiv \frac{1}{\sqrt{\left[n_{1}\right] !\left[n_{2}\right] ! \cdots\left[n_{i}\right] ! \cdots}}\left(a_{1}^{\dagger}\right)^{n_{1}}\left(a_{2}^{\dagger}\right)^{n_{2}} \cdots\left(a_{i}^{\dagger}\right)^{n_{i}} \cdots|0,0, \ldots\rangle
$$

are constructed as usual, so that

$$
\begin{gathered}
a_{i}^{\dagger}\left|n_{1}, \ldots, n_{i}, \ldots\right\rangle=\sqrt{\left[n_{i}+1\right]}\left|n_{1}, \ldots, n_{i}+1, \ldots\right\rangle, \\
a_{i}\left|n_{1}, \ldots, n_{i}, \ldots\right\rangle=\sqrt{\left[n_{i}\right]}\left|n_{1}, \ldots, n_{i}-1, \ldots\right\rangle, \\
\mathcal{N}_{i}\left|n_{1}, \ldots, n_{i}, \ldots\right\rangle=n_{i}\left|n_{1}, \ldots, n_{i}, \ldots\right\rangle .
\end{gathered}
$$

Here the bracketed notation

$$
[r]=\frac{1-q^{r}}{1-q}, \quad \text { along with } \quad[r] !=[1][2] \cdots[r-1][r], \quad[0] !=1,
$$


is used. The $q$-bracket $[A]$ for an operator $A$ is understood as a formal expression (formal series). At $q \rightarrow 1$, from $[r]$ and $[A]$ we recover $r$ and $A$, thus returning to the formulas for the standard bosonic oscillator. In what follows it will be assumed that

$$
-1 \leq q \leq 1
$$

for each such value of the deformation parameter $q$, the operators $a_{i}^{\dagger}, a_{i}$ are mutual conjugates.

In the generic case where $q \neq 1$, the bilinear $a_{i}^{\dagger} a_{i}$ does not equal the number operator $\mathcal{N}_{i}$ (as this is true for usual bosonic oscillators) but, instead,

$$
a_{i}^{\dagger} a_{i}=\left[\mathcal{N}_{i}\right] .
$$

The inverse of the latter relation is given by the formula [17]:

$$
\mathcal{N}_{i}=\sum_{s=1}^{\infty} \frac{(1-q)^{s}}{1-q^{s}}\left(a_{i}^{\dagger}\right)^{s} a_{i}^{s}
$$

expressing the number operator as a (formal) series of creation and annihilation operators.

\section{$\underline{\text { Type B }}$}

The $q$-oscillators of the second type are defined through the relations [18, 19]:

$$
\begin{gathered}
{\left[b_{i}, b_{j}\right]=\left[b_{i}^{\dagger}, b_{j}^{\dagger}\right]=0, \quad\left[N_{i}, b_{j}\right]=-\delta_{i j} b_{j}, \quad\left[N_{i}, b_{j}^{\dagger}\right]=\delta_{i j} b_{j}^{\dagger}, \quad\left[N_{i}, N_{j}\right]=0,} \\
b_{i} b_{j}^{\dagger}-q^{\delta_{i j}} b_{j}^{\dagger} b_{i}=\delta_{i j} q^{-N_{j}}, \quad b_{i} b_{j}^{\dagger}-q^{-\delta_{i j}} b_{j}^{\dagger} b_{i}=\delta_{i j} q^{N_{j}} .
\end{gathered}
$$

Again we have

$$
b_{i}^{\dagger} b_{i}=\left[N_{i}\right]
$$

where the notation for the $q$-bracket this time means:

$$
[r]=\frac{q^{r}-q^{-r}}{q-q^{-1}} .
$$

Formulas completely analogous to Eqs. (8)-(11) are valid also for the operators $b_{i}, b_{j}^{\dagger}$ if, instead of (12), we now use the definition (18) for $q$-brackets. Clearly, the equality $b_{i}^{\dagger} b_{i}=N_{i}$ holds only in the "no-deformation" limit of $q=1$. For consistency of the conjugation, it is required that either $q$ is real or

$$
q=\exp (i \theta), \quad 0 \leq \theta<2 \pi .
$$

In what follows we will consider the type " $\mathrm{A}$ " as well as the type "B" oscillators. For the type "B" oscillators the exponent form (19) will be adopted (compare with the symmetrization phase $\alpha$ in Eq. (5)). 


\section{$3 \quad$ Statistical $q$-distributions}

For the dynamical multi-pion or multi-kaon system, we consider the model of ideal gas of $q$-bosons (IQBG) taking the free (non-interacting) Hamiltonian in the form [20, 21]

$$
H=\sum_{i} \omega_{i} \mathcal{N}_{i}
$$

where $\omega_{i}=\sqrt{m^{2}+\mathbf{k}_{i}^{2}}, \mathcal{N}_{i}$ is defined as above, and subscript $i$ labels energy eigenvalues. It should be emphasized that among a large variety of possible choices of Hamiltonians, this is the unique truly non-interacting one, which possesses an additive spectrum. From now on, we assume that 3-momenta of particles take their values from a discrete set (i.e. the system is contained in a large finite box of volume $\sim L^{3}$ ).

As usual, basic statistical properties are obtained by evaluating thermal averages such as

$$
\langle A\rangle=\frac{\operatorname{Sp}(A \rho)}{\operatorname{Sp}(\rho)}, \quad \rho=e^{-\beta H},
$$

where $\beta=1 / T$ and the Boltzmann constant is set equal to 1 . The averaging here is taken with respect to the chosen Hamiltonian (20).

It is an easy task to calculate the quantity $\left\langle q^{\mathcal{N}_{i}}\right\rangle$, and to obtain

$$
\left\langle q^{\mathcal{N}_{i}}\right\rangle=\frac{e^{\beta \omega_{i}}-1}{e^{\beta \omega_{i}}-q} .
$$

From this we find the distribution function (recall that $q$ is from the interval $-1 \leq q \leq 1$ ):

$$
\left\langle a_{i}^{\dagger} a_{i}\right\rangle=\frac{1}{e^{\beta \omega_{i}}-q} .
$$

In the no-deformation limit $q \rightarrow 1$, this reduces to the Planck-Bose-Einstein distribution, as it should, since at $q=1$ we return to the standard system of bosonic commutation relations.

At $q=-1$ and $q=0$, the distributions we get coincide respectively with Fermi-Dirac and Maxwell-Boltzmann ones. It should be emphasized that this coincidence is rather formal: the defining relations (7) at $q=-1$ or $q=0$ differ from those for the system of fermions or the nonquantal (classical) system. The formal coincidence of Eq. (22) at $q=-1$ with a Fermi-Dirac distribution can be interpreted [22] as due to the impenetrability (the hard-core property) of such bosons. The difference with the system of genuine fermions lies in commuting (versus fermionic anticommuting) of non-coinciding modes at $q=-1$; see (7).

Let us now turn to the type "B" $q$-bosons. The Hamiltonian is chosen again as that of IQBG with the number operator defined in (16) and (17), i.e.,

$$
H=\sum_{i} \omega_{i} N_{i}
$$

Calculation of $\left\langle q^{ \pm N_{i}}\right\rangle$ yields

$$
\left\langle q^{ \pm N_{i}}\right\rangle=\frac{e^{\beta \omega_{i}}-1}{e^{\beta \omega_{i}}-q^{ \pm 1}} .
$$


From the relation

$$
\left\langle b_{i}^{\dagger} b_{i}\right\rangle=\frac{1}{e^{\beta \omega_{i}}-q}\left\langle q^{-N_{i}}\right\rangle
$$

or, equivalently, $\left\langle b_{i}^{\dagger} b_{i}\right\rangle=\left\langle a_{i}^{\dagger} a_{i}\right\rangle_{\mathrm{BE}}\left\langle q^{N_{i}}\right\rangle\left\langle q^{-N_{i}}\right\rangle$, we obtain the formula for the $q$-deformed distribution function (taking into account that $q+q^{-1}=[2]=2 \cos \theta$ ):

$$
\left\langle b_{i}^{\dagger} b_{i}\right\rangle=\frac{e^{\beta \omega_{i}}-1}{e^{2 \beta \omega_{i}}-2 \cos (\theta) e^{\beta \omega_{i}}+1} .
$$

Note that, although the deformation parameter $q$ is chosen in a particular complex form, see (19), the explicit expression for the $q$-distribution function turns out to be real, owing to its specific dependence on $q$ through the combination $q+q^{-1}$.

The shape of the function $f(k) \equiv\left\langle b^{\dagger} b\right\rangle(k)$ from (25) corresponding to the gas of pions modelled by IQBG is picturef in Fig. 1 (curve II). For comparison, the standard Bose-Einstein distribution function (curve I) and the classical Maxwell-Boltzmann one (curve III) are also presented in the same figure. As is clearly seen, the $q$-deformed distribution function lies completely in between the other two curves, thus demonstrating] that the deviation of the $q$-distribution (25) from the quantum Bose-Einstein distribution goes in the "right direction", towards the classical Maxwell-Boltzmann one.

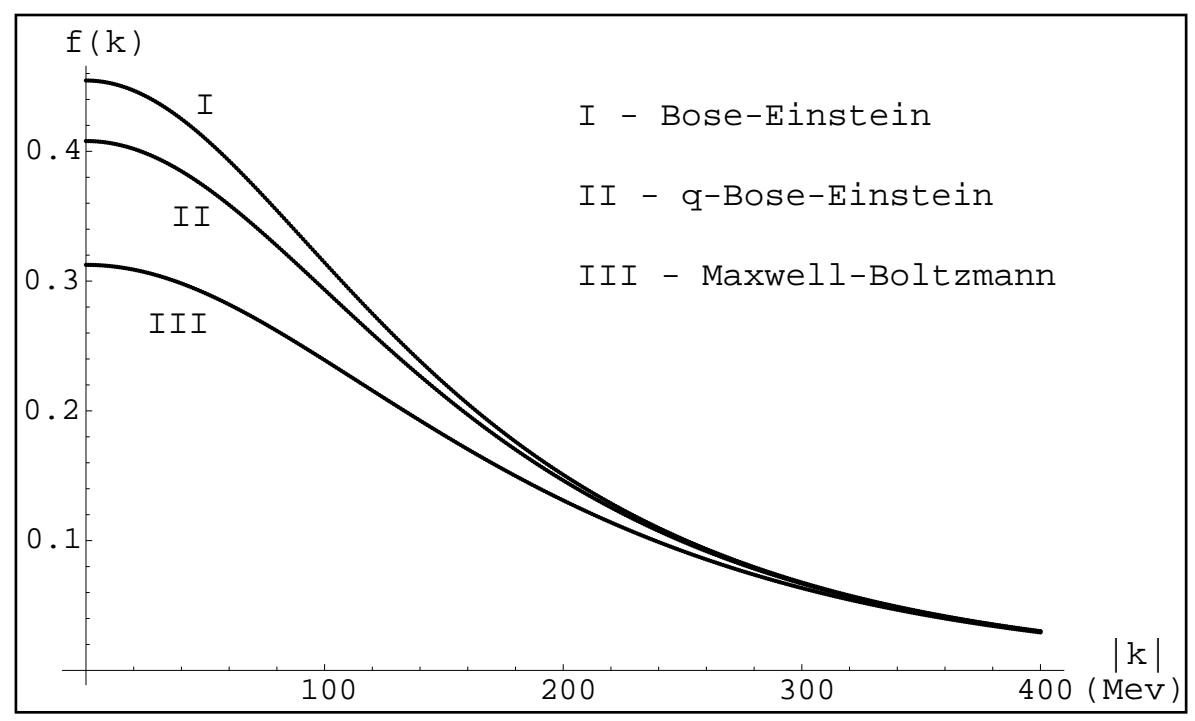

Figure 1: The $q$-distribution function (25) versus momentum (curve II), in comparison with the quantum Bose-Einstein (curve I) and classical Maxwell-Boltzmann (curve III) distributions. The inputs are: $T=120 \mathrm{MeV}, m=m_{\pi}$; curve II corresponds to the deformation angle $\theta=24^{\circ}$.

\footnotetext{
${ }^{4}$ The (isotriplet-averaged) pion mass $m\left(\pi^{ \pm, 0}\right)=139.57 \mathrm{MeV}$ and the temperature $T=120 \mathrm{MeV}$ are taken as inputs. The deformation value, encoded in $\cos \theta$, is fixed to be $\theta=24^{\circ}$.

${ }^{5}$ The same is true also for the (apparently more simple) $q$-distribution (22) of the type "A" $q$-bosons.
} 
Analogous curves for $q$-distribution functions, with similar properties, can be given for other fixed data. Let us remark that, in the case of kaons, because of their larger mass and higher empirical value of the intercept $\lambda \simeq 0.88$ (which corresponds to the smaller deformation in our model), such a curve should lie significantly closer to that of the Bose-Einstein distribution.

It is worth noting that the $q$-distribution functions (22) and (25) already appeared (in the context of thermal field theory) in [21]. We give them here for completeness of exposition, since they are connected with new results to be described in the next section.

\section{Two-particle correlations of $q$-bosons}

Let us now turn to the issue of two-particle correlations. From the easily verifiable identity

$$
a_{i}^{\dagger} a_{j}^{\dagger} a_{k} a_{l}-q^{-\delta_{i k}-\delta_{i l}} a_{j}^{\dagger} a_{k} a_{l} a_{i}^{\dagger}=\left[a_{i}^{\dagger}, a_{j}^{\dagger}\right] a_{k} a_{l}+a_{j}^{\dagger}\left[a_{i}^{\dagger}, a_{k}\right]_{q^{-\delta_{i k}}} a_{l}+q^{-\delta_{i k}} a_{j}^{\dagger} a_{k}\left[a_{i}^{\dagger}, a_{l}\right]_{q^{-\delta_{i l}}},
$$

by taking thermal averages, we find

$$
\left\langle a_{i}^{\dagger} a_{j}^{\dagger} a_{k} a_{l}\right\rangle=\frac{e^{\beta \omega_{i}}-q}{q^{1-\delta_{i k}-\delta_{i l}} e^{\beta \omega_{i}}-q}\left(\left\langle a_{j}^{\dagger} a_{l}\right\rangle\left\langle a_{i}^{\dagger} a_{k}\right\rangle+q^{-\delta_{i j}}\left\langle a_{j}^{\dagger} a_{k}\right\rangle\left\langle a_{i}^{\dagger} a_{l}\right\rangle\right) .
$$

With coinciding modes 0 , this leads to the formula

$$
\left\langle a_{i}^{\dagger} a_{i}^{\dagger} a_{i} a_{i}\right\rangle=\frac{1+q}{\left(e^{\beta \omega_{i}}-q\right)\left(e^{\beta \omega_{i}}-q^{2}\right)} .
$$

From the last relation and distribution (22), the ratio under question follows:

$$
\tilde{\lambda}_{i}=\frac{\left\langle a_{i}^{\dagger} a_{i}^{\dagger} a_{i} a_{i}\right\rangle}{\left\langle a_{i}^{\dagger} a_{i}\right\rangle^{2}}=\frac{(1+q)\left(e^{\beta \omega_{i}}-q\right)}{e^{\beta \omega_{i}}-q^{2}}
$$

This constitutes one of our main results. For convenience, let us set

$$
\tilde{\lambda}=1+\lambda \quad \text { with } \quad \lambda=q \frac{e^{\beta \omega}-1}{e^{\beta \omega}-q^{2}}
$$

The quantity $\lambda$ can be directly confronted with empirical data. Note that in the non-deformed limit $q \rightarrow 1$ the value $\lambda_{\mathrm{BE}}=1$, proper for Bose-Einstein statistics, is correctly reproduced from Eq. (28). This, obviously, corresponds to the Bose-Einstein distribution contained in (22) if $q \rightarrow$ At the value $\lambda_{\mathrm{FD}}=-1$ proper for the Fermi-Dirac statistics respectively, although the defining relations are not exactly those of the fermionic system (rather, the hard core bosons [22]). Finally, at $q=0$ we get $\lambda=0$, which coincides with the analogous fact for the case of purely classical description (complete absence of quantum effects due to identical particles). The three different cases are clearly seen in Fig. 2 as the only three points where all the different curves

\footnotetext{
${ }^{6}$ Recall that $\mathbf{K} \equiv \frac{1}{2}\left(\mathbf{k}_{a}+\mathbf{k}_{b}\right), \mathbf{p} \equiv \mathbf{k}_{a}-\mathbf{k}_{b}$; in the case $\mathbf{p}=0, \mathbf{K}=\mathbf{k}_{a}=\mathbf{k}_{b}$.
} 


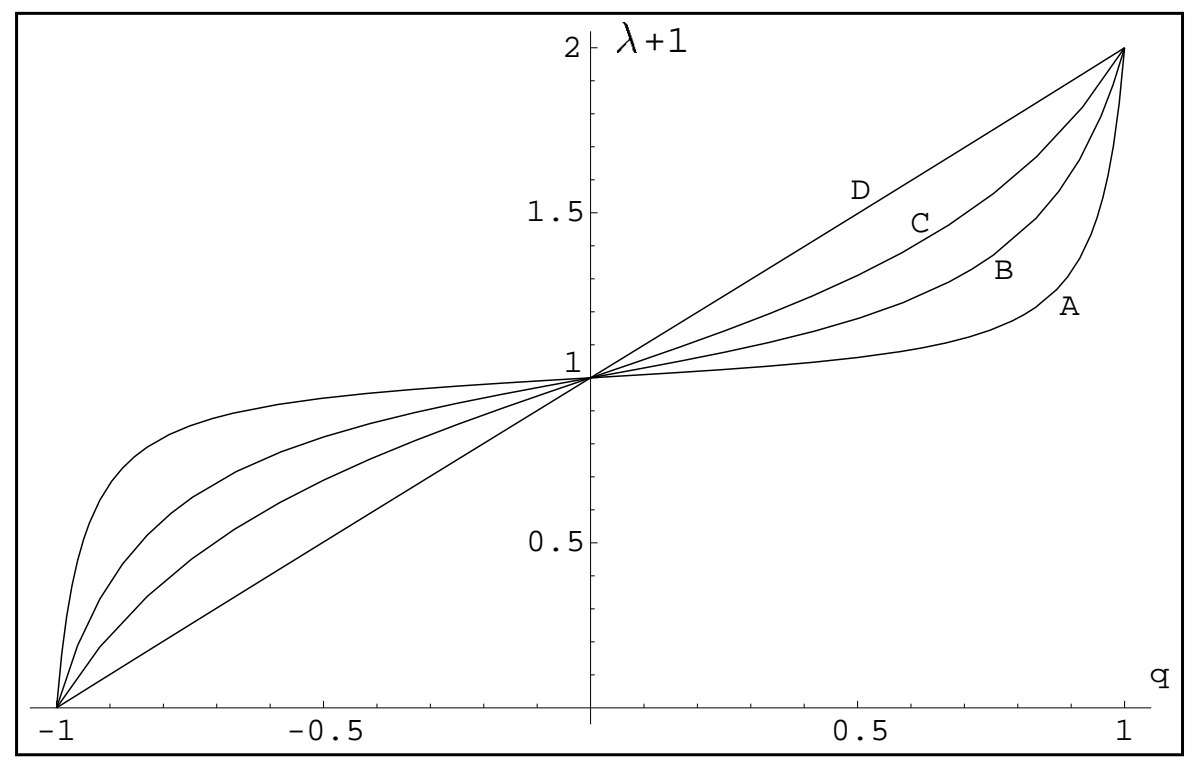

Figure 2: Intercept $\lambda$ versus deformation parameter $q$, as given by Eq. (28). The curves A, $\mathrm{B}, \mathrm{C}$ and $\mathrm{D}$ correspond to the values $w_{A}=0.1, w_{B}=0.35, w_{C}=0.8$ and $w_{D}=5.0$ of the dimensionless variable $w \equiv \beta \omega$.

(the continuum parametrized by $w=\beta \omega)$ merge and, thus, the dependence on momentum and/or temperature disappears. From the continuum of curves, there exists a unique limiting (asymptotic) one $\tilde{\lambda}=1+q$ (or $\lambda=q$ ), which corresponds to the limit $w \rightarrow \infty$ (i.e. to zero temperature or infinite momentum). Conversely, for very large temperature such that $w \rightarrow 0$, the curve goes over into the step-shaped function

$$
\tilde{\lambda}=\left\{\begin{array}{l}
0, \quad q=-1, \\
1, \quad-1<q<1, \\
2, \quad q=1,
\end{array}\right.
$$

with the constant $\lambda=0$ for each fixed value of $q$ except for the endpoints $q=1$ and $q=-1$.

We find now the formula describing two-particle correlations (at identical momenta), which corresponds to Biedenharn-Macfarlane q-oscillators, see Eq. (16). From the relation

$$
\left\langle b_{i}^{\dagger} b_{i}^{\dagger} b_{i} b_{i}\right\rangle-q^{2}\left\langle b_{i}^{\dagger} b_{i} b_{i} b_{i}^{\dagger}\right\rangle=-\left\langle b_{i}^{\dagger} b_{i} q^{N_{i}}\right\rangle\left(1+q^{2}\right),
$$

which is valid just for coinciding modes (i.e. equal momenta), we immediately get

$$
\left\langle b_{i}^{\dagger} b_{i}^{\dagger} b_{i} b_{i}\right\rangle=\frac{1+q^{2}}{q^{2} e^{\beta \omega_{i}}-1}\left\langle b_{i}^{\dagger} b_{i} q^{N_{i}}\right\rangle .
$$

The thermal average in the r.h.s. can be easily evaluated to yield $\left\langle b_{i}^{\dagger} b_{i} q^{N_{i}}\right\rangle=q /\left(e^{\beta \omega_{i}}-q^{2}\right)$. 
Taking this into account, we find the expression for two-particle distribution, namely

$$
\left\langle b_{i}^{\dagger} b_{i}^{\dagger} b_{i} b_{i}\right\rangle=\frac{2 \cos \theta}{e^{2 \beta \omega_{i}}-2 \cos (2 \theta) e^{\beta \omega_{i}}+1} .
$$

From that, the desired formula for (the intercept of) two-particle correlations finally results in:

$$
\tilde{\lambda}_{i} \equiv \lambda_{i}+1=\frac{\left\langle b_{i}^{\dagger} b_{i}^{\dagger} b_{i} b_{i}\right\rangle}{\left(\left\langle b_{i}^{\dagger} b_{i}\right\rangle\right)^{2}}=\frac{2 \cos \theta\left(t_{i}+1-\cos \theta\right)^{2}}{t_{i}^{2}+2\left(1-\cos ^{2} \theta\right) t_{i}},
$$

where $t_{i}=\cosh \left(\beta \omega_{i}\right)-1$. Note that both (29) and (30) are real functions (as they should) since, like (25), they both depend on the complex $q$-parameter (19) through the combination $\frac{1}{2}\left(q+q^{-1}\right)=\cos \theta$.

In the rest of this section, we extract some useful information contained in Eq. (30). Solving this equation with respect to $\cos \theta$ at a fixed value $\lambda=\bar{\lambda}$, we obtain the deformatin angle as the function: $\theta=\theta(\bar{\lambda}, \mathbf{K}, T, m)$.

Figure 3 is used to illustrate main properties of the intercept (correlation strength) $\lambda$ treated from the standpoint of $q$-deformation, that is, on the base of Eq. (30). First, let us note that the continuum of curves $\tilde{\lambda}=\tilde{\lambda}(\cos \theta)$ parametrized by $w=\beta \omega$ divides into three different classes ("subcontinua") given by three intervals of the parameter: (i) $0<w \leq w_{0}$, (ii) $w_{0}<w<w_{0}^{\prime}$, and (iii) $w_{0}^{\prime} \leq w<\infty$. Here, the two "critical" values $w_{0}=w_{B} \simeq 0.481$ and $w_{0}^{\prime}=w_{D} \simeq 0.696$ are singled out (curves B and D respectively). The curves A, C, and E are typical representatives of the classes (i),(ii) and (iii). All the curves from classes (i), (ii) possess two extrema, the minimum being to the left of the maximum (the curve D is unique since its extrema degenerate, coinciding with the point of inflection). This fact enables us to define naturally "the range of small deformations" - the interval $I_{\text {small }}$ for the variable $\theta$ : from $\theta=0$ (no deformation) to the value yielding minimal $\lambda, \lambda_{\min } \approx 0.33$, implied by the "critical" value $w_{0}=w_{D}$. It is seen that on the interval $I_{\text {small }}$ the intercept $\lambda$ monotonically decreases with increasing of $\theta$ (or $\left.1-\cos \theta\right)$, the strength of deformation. On the contrary, each curve from the third class is monotonic as a whole and, thus, there is no criterion (no peculiar point), which would naturally separate "small" deformations from "large" ones.

In this paper we deal only with classes (ii) and (iii) since all their curves at $\theta \neq 0$ lie below the straight line $\tilde{\lambda}=2$ - the largest possible correlation attainable in the Bose-Einstein case (note that curve $\mathrm{B}$ contains, besides $\theta=0$, just a single point at a certain value of $1-\cos \theta$, where the value $\tilde{\lambda}=2$ is also attained). Moreover, for the (ii)-type curves, we restrict ourselves to $I_{\text {small }}$, ignoring "moderately large" deformations (between min. and max.), for which the behaviour of $\lambda=\lambda(\theta)$ is opposite to that for $I_{\text {small, }}$ as well as very large ones (to the right of the maximum). Unlike these two regular classes, the class (i) consists of "irregular" curves: for each such curve there exist $q$-deformations that generate correlation strengths exceeding the maximal possible one $\tilde{\lambda}=2$. Therefore, we discard the class (i), at least at this stage.

Finally, let us discuss special values of the physical variables $T,|\mathbf{K}|$, which provide the peculiar values $w_{0}=w_{B} \simeq 0.481$ and $w_{0}^{\prime}=w_{D} \simeq 0.696$ (recall that $w=\sqrt{m^{2}+\mathbf{K}^{2}} / T$ ). With $m\left(\pi^{ \pm, 0}\right)=139.57 \mathrm{MeV}$ and lowest mean momentum of the pion pair fixed to be $|\mathbf{K}|=0$, we get two bounded from below values for the temperature: $T_{0}=290.0 \mathrm{MeV}$ and $T_{0}^{\prime}=200.5 \mathrm{MeV}$. 


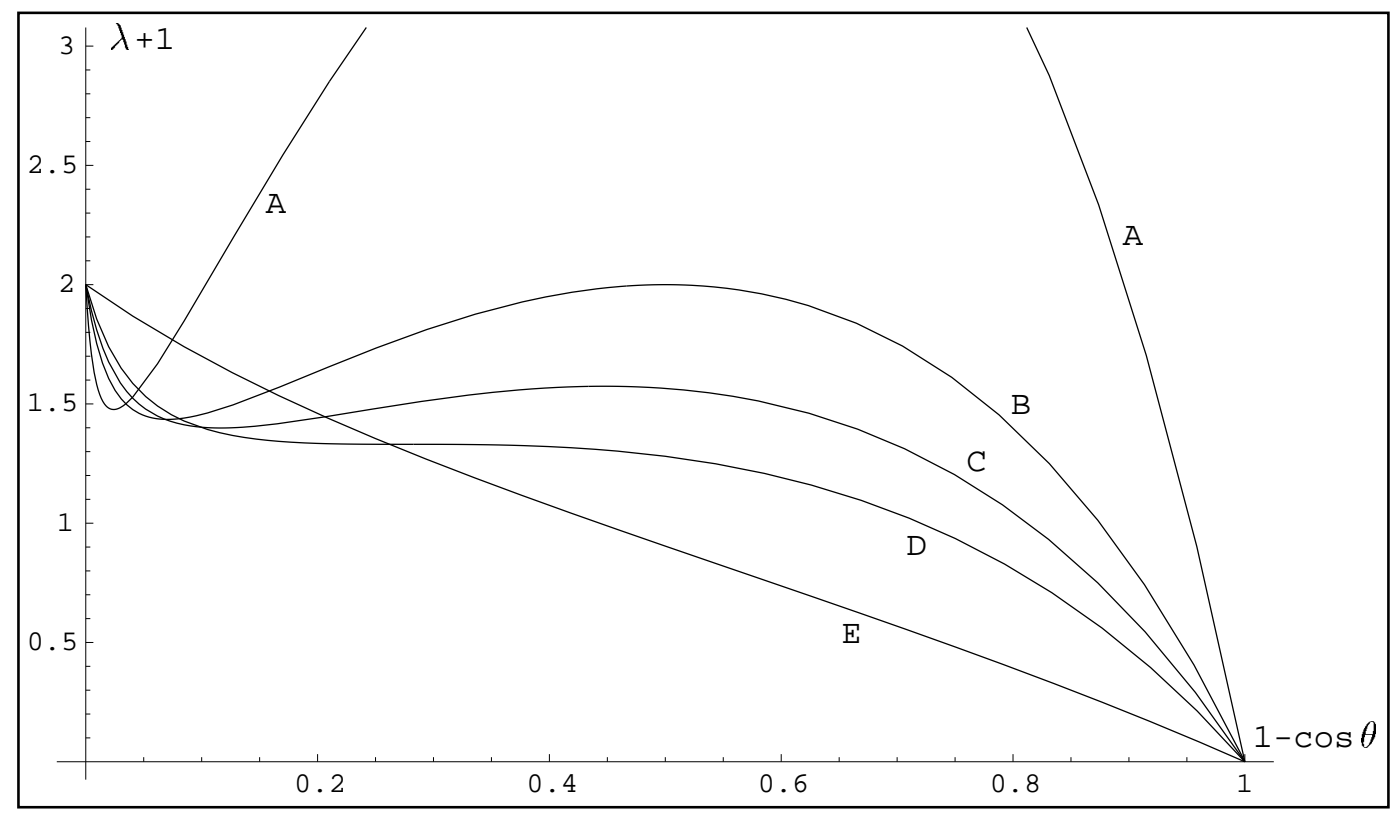

Figure 3: Intercept $\lambda$ versus deformation given by $\cos \theta$, see Eq. (30). The curves A,B,C,D and $\mathrm{E}$ correspond to the values $w_{A}=0.3, w_{B}=0.481, w_{C}=0.58, w_{D}=0.696$ and $w_{E}=2.0$ of the dimensionless variable $w \equiv \beta \omega$.

It is interesting to compare these data with that for the typical curve from class (iii). Namely, at $w=w_{E}=2.0$ (the curve E) for pions of this same lowest momentum we get the limiting temperature $T_{E}=69.8 \mathrm{MeV}$.

\section{$5 \quad$ Discussion and outlook}

The main purpose of theoretical approaches to RHIC is to find an adequate description for the non-equilibrium state formed during the collision. On this way, the $q$-boson techniques enables us to treat the non-stationary hot and dense matter effectively as a "noninteracting ideal gas". To deal with $q$-bosons, it is necessary to determine the $q$-parameter that corresponds to the actual state of the hot medium. We propose a way of extracting from the two-particle correlations a useful information concerning $q$, and develop an effective picture of the two-pion (-kaon) spectra in RHIC. According to our results, the measured deviation from unity of the intercept $\lambda$ is interpreted as the manifestation of $q$-boson properties of the pion system created in RHIC.

From Eqs. (28) and (30), one can express the quantities encoding the deformation as: $q=q(\lambda, \mathbf{K}, T, m)$ and $\theta=\theta(\lambda, \mathbf{K}, T, m)$. In the characteristic limits of temperature and momentum, our model exhibits a remarkable feature, valid for both types of exploited q-oscillators:

- For very low temperature at fixed momenta, or very large momenta at fixed temperature (i.e. at $w \rightarrow \infty$, compare with curve D in Fig. 2 and curve E in Fig. 3), we come to the equality

$$
q=\lambda \quad(T \rightarrow 0 \quad \text { or } \quad|\mathbf{K}| \rightarrow \infty)
$$


with the type " $A$ " oscillators, and to the equality

$$
2 \cos \theta=\lambda+1 \quad(T \rightarrow 0 \quad \text { or } \quad|\mathbf{K}| \rightarrow \infty)
$$

for the type " $B$ " oscillators. This implies the unified direct connection $\lambda \leftrightarrow q$, namely $\tilde{\lambda}=[2]$, for both types "A" and "B".

On the other hand, finite temperature and momenta become non-trivially involved (especially in the case of type "B" $q$-bosons) in the relation between $\lambda$ and the deformation parameter q, see Eqs. (28), (30) as well as Figs. 2, 3.

- Equation (30) implies dependence of the intercept $\lambda$ on the pair mean momentum $\mathbf{K}$ for fixed values of the deformation angle $\theta$, temperature and particle mass, since $\lambda=\lambda(\theta, \mathbf{K}, T, m)$. In Fig. 4, we present this dependence exemplifying it, for $T=120 \mathrm{MeV}$ and $m=m_{\pi}$, with four curves which correspond to the values $4^{\circ}, 9^{\circ}, 15^{\circ}$ and $24^{\circ}$ of the deformation angle $\theta$. Each curve tends to its own asymptote given by Eq. (32). The apparent variability of $\lambda$ with varying $|\mathbf{K}|$ (for a fixed deformation) is one of the consequences of our model, and can be viewed both as an interesting prediction and as a check-point for this approach.

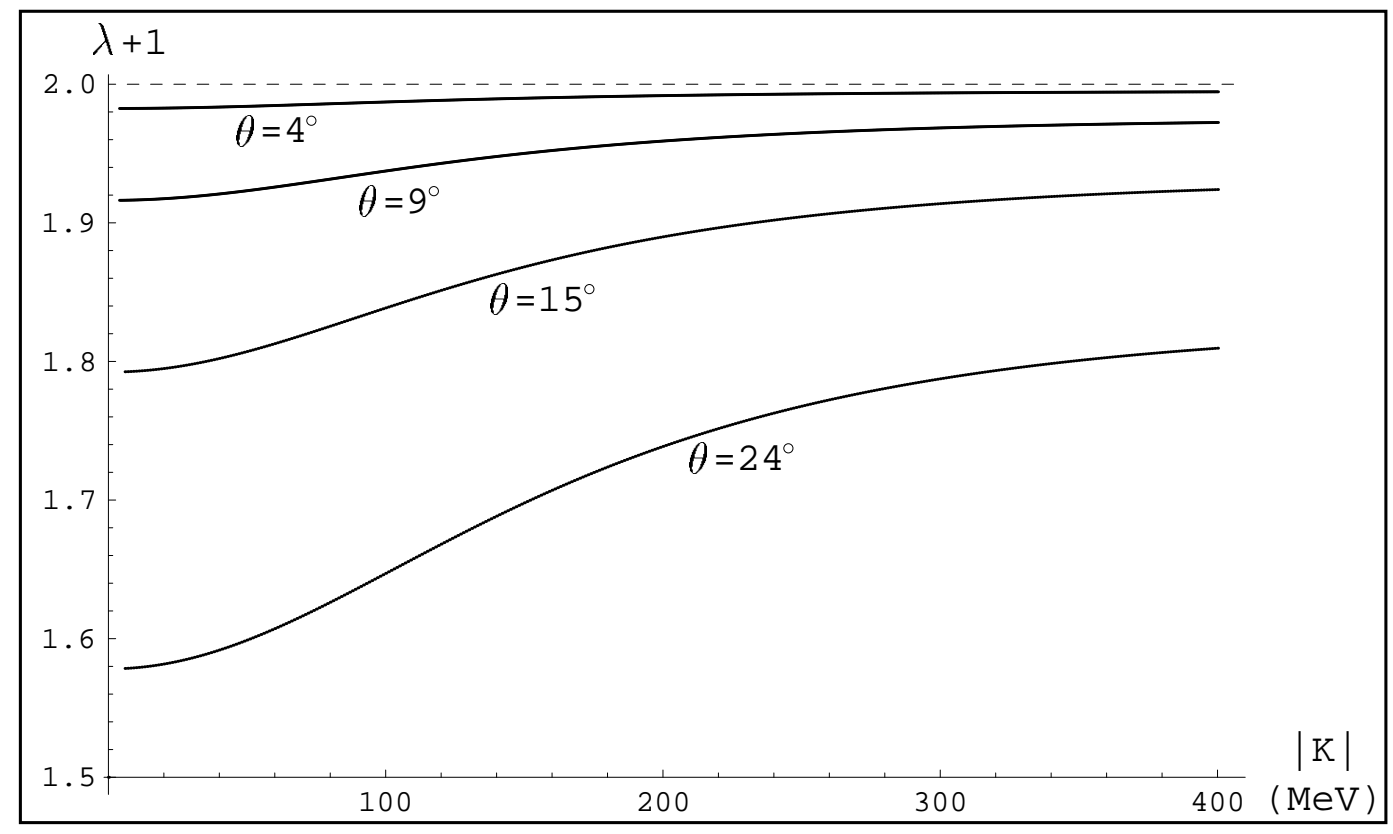

Figure 4: Dependence of the intercept $\lambda$ on the pion momentum $|\mathbf{K}|$ for some values of $\theta$. The temperature is fixed to be $T=120 \mathrm{MeV} ; m=m_{\pi}$.

In [16], it was argued that the quon-based description applied to pions participating in the decay $K_{\mathrm{L}} \rightarrow \pi^{+} \pi^{-}$, can give a complete account of this process (instead of $C P$-violation, as commonly accepted cause). Moreover, this sets a bound on the "strength" of the deformation, namely $1-q \leq 10^{-6}$. On the contrary, within the proposed approach to multiparticle correlations (as occurred in the HBT interferometry) based on viewing the identical pions as $q$-bosons, the two-pion correlations imply the employment of significantly more developed (strength of) $q$-deformation. Namely, in our implementation of the $q$-Bose gas picture, the empirical data are interpreted as corresponding to the strength $\simeq 0.2--0.6$ of $q$-deformation. 
The approach proposed in this paper, we hope, opens interesting new perspectives for further research in this direction. Three-particle correlations as well as fermion-fermion(-fermion) ones are the topics to be studied next using the developed " $q$-techniques".

\section{Acknowledgements}

D.A. acknowledges many helpful and instructive discussions with U. Heinz. The work of two of us (A.G. and N.I.) was partially supported by the Award No. UP1-309 of the U.S. Research and Development Foundation for the Independent States of the Former Soviet Union (CRDF), and by the Ukrainian DFFD Grant 1.4/206.

\section{APPENDIX}

In the appendix we consider the two-particle quantum statistical correlations when the final state interactions of the detected particles are neglected. This phenomenon is visualized most transparently on the basis of standard quantum mechanics in the non-relativistic case. Meanwhile, using the presented scheme, the relativistic picture can also be considered. Moreover, this approach allows us to take into consideration the final state interactions as well [4].

The probability to register two particles, with definite asymptotic momenta $\mathbf{p}_{a}$ and $\mathbf{p}_{b}$, which are created in the relativistic heavy-ion collisions is usually compared with the probability to register two particles of the same momentum independently. For that reason, we begin by considering the single-particle spectrum.

\section{Single-particle cross section}

Let us consider the single-particle state $\psi_{\gamma}$ of a particle emitted by the source. Its propagation to the detector is governed by the Schrödinger equation

$$
i \frac{\partial \psi_{\gamma}(\mathbf{x}, t)}{\partial t}=\hat{h}(\mathbf{x}) \psi_{\gamma}(\mathbf{x}, t), \quad \text { where } \quad \hat{h}(\mathbf{x})=-\frac{1}{2 m} \nabla^{2} .
$$

The index $\gamma$ denotes a complete set of 1-particle quantum numbers. (In a basis of wave packets, these could contain the centers $\mathbf{X}$ of the wave packets of the particles at their freeze-out times t.) Equation (33) is solved by

$$
\psi_{\gamma}\left(\mathbf{x}, t, t_{0}\right)=e^{-i \hat{h}(\mathbf{x})\left(t-t_{0}\right)} \psi_{\gamma}\left(\mathbf{x}, t_{0}\right)
$$

in terms of the single-particle wave function at some initial time $t_{0}$. We will assume that the detector measures asymptotic momentum eigenstates, i.e. that it acts by projecting the emitted 1-particle state onto

$$
\phi_{\mathbf{p}}^{\text {out }}(\mathbf{x}, t)=e^{i(\mathbf{p} \cdot \mathbf{x}-\omega(\mathbf{p}) t)}
$$


where $\omega(\mathbf{p})=\mathbf{p}^{2} / 2 m$ is the energy of the particle. The measured single-particle momentum amplitude is then

$$
A_{\gamma}\left(\mathbf{p}, t_{0}\right)=\lim _{t \rightarrow \infty} \int d^{3} x \phi_{\mathbf{p}}^{\text {out }, *}(\mathbf{x}, t) \psi_{\gamma}\left(\mathbf{x}, t, t_{0}\right) .
$$

Using the time evolution equation (34), this can be expressed in terms of the emitted singleparticle wave function $\psi_{\gamma}$ at earlier times as

$$
\begin{aligned}
A_{\gamma}\left(\mathbf{p}, t_{0}\right) & =\lim _{t \rightarrow \infty} \int d^{3} x\left[e^{-i \hat{h}(\mathbf{x})\left(t_{0}-t\right)} \phi_{\mathbf{p}}^{\text {out }}(\mathbf{x}, t)\right]^{*} \psi_{\gamma}\left(\mathbf{x}, t_{0}\right) \\
& =\int d^{3} x e^{i \omega(\mathbf{p}) t_{0}-i \mathbf{p} \cdot \mathbf{x}} \psi_{\gamma}\left(\mathbf{x}, t_{0}\right)
\end{aligned}
$$

This expression means that the momentum amplitude, as it should be, is an on-shell Fourier transformation of the emitted wave at emission times. It is worth noting that this is not the case when, after emission, a particle is subject to final state interactions.

The single-particle probability is obtained by averaging (37) and its complex conjugate using the density matrix defining the source. This density matrix is characterized by a probability distribution for the single-particle quantum numbers $\gamma$. We write

$$
P_{1}(\mathbf{p})=\overline{\left\langle\left|A_{\gamma}\left(\mathbf{p}, t_{0}\right)\right|^{2}\right\rangle_{\gamma}}=\sum_{\gamma \gamma^{\prime}} \int d t_{0} d t_{0}^{\prime} \rho_{\gamma, \gamma^{\prime}} A_{\gamma^{\prime}}\left(\mathbf{p}, t_{0}^{\prime}\right) A_{\gamma}^{*}\left(\mathbf{p}, t_{0}\right),
$$

where the anglular brackets mean averaging over source quantum numbers $\gamma$; the overline means averaging over emission times and is detailed in the r.h.s. Inserting (37) into (38) yields

$$
P_{1}(\mathbf{p})=\int d^{4} x d^{4} x^{\prime} e^{i p \cdot\left(x-x^{\prime}\right)} \sum_{\gamma, \gamma^{\prime}} \rho_{\gamma \gamma^{\prime}} \psi_{\gamma}(x) \psi_{\gamma^{\prime}}^{*}\left(x^{\prime}\right),
$$

where $p=(\omega(\mathbf{p}), \mathbf{p})$. Let us introduce new time and position space variables

$$
Y=\frac{1}{2}\left(x+x^{\prime}\right), \quad y=x-x^{\prime} .
$$

We now define the single particle Wigner density $S(X, K)$ of the source as

$$
S(Y, K)=\int d^{4} y e^{i K \cdot y} \sum_{\gamma, \gamma^{\prime}} \rho_{\gamma \gamma^{\prime}} \psi_{\gamma}\left(Y+\frac{y}{2}\right) \psi_{\gamma^{\prime}}^{*}\left(Y-\frac{y}{2}\right)
$$

Using the hermiticity of the source density matrix $\rho_{\gamma \gamma^{\prime}}$ it is easily shown that $S(Y, K)$ is real. Thus, we come to the expression for the single-particle spectrum, which employs the source function:

$$
P_{1}(\mathbf{p})=\int d^{4} x S(x, p)
$$

where the integration goes over emission times. 


\section{Two-particle quantum statistical correlations without final state interactions}

Let us consider a two-particle state $\psi_{\gamma}$ emitted by the source. Its propagation to the detector is governed by the Schrödinger equation

$$
i \frac{\partial \psi_{\gamma}\left(\mathbf{x}_{a}, \mathbf{x}_{b}, t\right)}{\partial t}=\hat{H}\left(\mathbf{x}_{a}, \mathbf{x}_{b}\right) \psi_{\gamma}\left(\mathbf{x}_{a}, \mathbf{x}_{b}, t\right)
$$

where $\hat{H}\left(\mathbf{x}_{a}, \mathbf{x}_{b}\right)=\hat{h}\left(\mathbf{x}_{a}\right)+\hat{h}\left(\mathbf{x}_{b}\right)$. The index $\gamma$ denotes a complete set of 2-particle quantum numbers. (In a basis of products of two wave packets these could contain the centers $\mathbf{X}_{a}, \mathbf{X}_{b}$ of the wave packets of the two particles at their freeze-out times $t_{a}, t_{b}$, respectively.) Equation (43) is solved by

$$
\psi_{\gamma}\left(\mathbf{x}_{a}, \mathbf{x}_{b}, t\right)=e^{-i \hat{H}\left(\mathbf{x}_{a}, \mathbf{x}_{b}\right)\left(t-t_{0}\right)} \psi_{\gamma}\left(\mathbf{x}_{a}, \mathbf{x}_{b}, t_{0}\right)
$$

in terms of the two-particle wave function at some initial time $t_{0}$. We will assume that the detector measures asymptotic momentum eigenstates, i.e. that it acts by projecting the emitted 2-particle state onto

$$
\phi_{\mathbf{p}_{a}, \mathbf{p}_{b}}^{\text {out }}\left(\mathbf{x}_{a}, \mathbf{x}_{b}, t\right)=e^{i\left(\mathbf{p}_{a} \cdot \mathbf{x}_{a}-\omega_{a} t\right)} e^{i\left(\mathbf{p}_{b} \cdot \mathbf{x}_{b}-\omega_{b} t\right)}
$$

where $\omega_{a, b}=\mathbf{p}_{a, b}^{2} / 2 m^{2}$. We will only consider the case of pairs of identical particles, $m_{a}=m_{b}=$ $m$. The measured two-particle momentum amplitude is then

$$
A_{\gamma}\left(\mathbf{p}_{a}, \mathbf{p}_{b}\right)=\lim _{t \rightarrow \infty} \int d^{3} x_{a} d^{3} x_{b} \phi_{\mathbf{p}_{a}, \mathbf{p}_{b}}^{\text {out }}\left(\mathbf{x}_{a}, \mathbf{x}_{b}, t\right) \psi_{\gamma}\left(\mathbf{x}_{a}, \mathbf{x}_{b}, t\right)
$$

If we consider identical particles, then the two-particle wave function $\psi_{\gamma}\left(\mathbf{x}_{a}, \mathbf{x}_{b}, t\right)$ should be symmetrized (antisymmetrized). Writing this explicitly, we obtain

$$
A_{\gamma}\left(\mathbf{p}_{a}, \mathbf{p}_{b}\right)=\lim _{t \rightarrow \infty} \frac{1}{\sqrt{2}} \int d^{3} x_{a} d^{3} x_{b} \phi_{\mathbf{p}_{a}, \mathbf{p}_{b}}^{\text {out }}\left(\mathbf{x}_{a}, \mathbf{x}_{b}, t\right)\left[\psi_{\gamma}\left(\mathbf{x}_{a}, \mathbf{x}_{b}, t\right)+e^{i \alpha} \psi_{\gamma}\left(\mathbf{x}_{b}, \mathbf{x}_{a}, t\right)\right]
$$

where $\alpha=0$ ( $\alpha=\pi)$ for identical bosons (fermions). Relabelling the variables of integration in the second term on the r.h.s. of this equation as $\mathbf{x}_{a} \rightarrow \mathbf{x}_{b}$ and $\mathbf{x}_{b} \rightarrow \mathbf{x}_{a}$, we come to the expression:

$$
\begin{gathered}
A_{\gamma}\left(\mathbf{p}_{a}, \mathbf{p}_{b}\right)=\lim _{t \rightarrow \infty} \frac{1}{\sqrt{2}} \int d^{3} x_{a} d^{3} x_{b}\left[e^{i\left(\mathbf{p}_{a} \cdot \mathbf{x}_{a}-\omega_{a} t\right)} e^{i\left(\mathbf{p}_{b} \cdot \mathbf{x}_{b}-\omega_{b} t\right)}+e^{i \alpha} e^{i\left(\mathbf{p}_{a} \cdot \mathbf{x}_{b}-\omega_{a} t\right)} e^{i\left(\mathbf{p}_{b} \cdot \mathbf{x}_{a}-\omega_{b} t\right)}\right]^{*} \\
\times \psi_{\gamma}\left(\mathbf{x}_{a}, \mathbf{x}_{b}, t\right)
\end{gathered}
$$

This simple algebra results in the evident conclusion that it does not matter which wave function should be symmetrized (antisymmetrized) at asymptotic time $t$ : two-particle wave function $\psi_{\gamma}\left(\mathbf{x}_{a}, \mathbf{x}_{b}, t\right)$ or out-state wave function. We show below that the same holds for emission times.

Using the evolution operator (see (44)), Eq. (46) can be expressed in terms of the twoparticle wave function $\psi_{\gamma}$ emitted at earlier times as

$$
A_{\gamma}\left(\mathbf{p}_{a}, \mathbf{p}_{b}\right)=\lim _{t \rightarrow \infty} \int d^{3} x_{a} d^{3} x_{b}\left[e^{-i \hat{H}\left(\mathbf{x}_{a}, \mathbf{x}_{b}\right)\left(t_{0}-t\right)} \phi_{\mathbf{p}_{a}, \mathbf{p}_{b}}^{\text {out }}\left(\mathbf{x}_{a}, \mathbf{x}_{b}, t\right)\right]^{*} \psi_{\gamma}\left(\mathbf{x}_{a}, \mathbf{x}_{b}, t_{0}\right)
$$


This is correct for all times $t_{0} \geq \max \left[t_{a}, t_{b}\right]$, where $t_{a, b}$ are the freeze-out times for the two particles. Note that the time evolution operator has been shifted (by Hermitian inversion of the unitary evolution operator) from the emitted two-particle state with arbitrary quantum numbers $\gamma$ to the two-particle momentum eigenstate $\phi_{\mathbf{p}_{a}, \mathbf{p}_{b}}^{\text {out }}\left(\mathbf{x}_{a}, \mathbf{x}_{b}, t\right)$ which is thereby transformed into a plane wave at time $t_{0}$. It should be pointed, that such an evolution of the out-state from $t=\infty$ to $t=t_{0}$ if one includes the two-particle interaction into the Hamiltonian $\hat{H}\left(\mathbf{x}_{a}, \mathbf{x}_{b}\right)$, would bring a distorted wave at time $t_{0}$ instead of the plane one [4].

We assume that the two particles are emitted independently, implying that, at some freezeout time $t_{a}$, the two-particle wave function $\psi_{\gamma}\left(\mathbf{x}_{a}, \mathbf{x}_{b}, t\right)$ factorizes

$$
\psi_{\gamma}\left(\mathbf{x}_{a}, \mathbf{x}_{b}, t_{a}\right)=\frac{1}{\sqrt{2}}\left[\psi_{\gamma_{a}}\left(\mathbf{x}_{a}, t_{a}\right) \psi_{\gamma_{b}}\left(\mathbf{x}_{b}, t_{a}\right)+e^{i \alpha} \psi_{\gamma_{a}}\left(\mathbf{x}_{b}, t_{a}\right) \psi_{\gamma_{b}}\left(\mathbf{x}_{a}, t_{a}\right)\right]
$$

The indices $\gamma_{a}, \gamma_{b}$ on the 1-particle wave functions now label complete sets of 1-particle quantum numbers. Time $t_{a}$ is the emission time of the latest emitted particle. At this time the first emitted particle of the pair has already propagated for a time $t_{a}-t_{b}$ if it was emitted at $t_{b}<t_{a}$. During this time the first emitted particle cannot "see" the second particle as a separate entity, but only as part of the remaining fireball. That is why the two-particle symmetrization (antisymmetrization) can be effectively extracted from many-particle symmetrization (antisymmetrization) after emission of the second particle. In other words a factorization of the two-particle wave function from the many-particle wave function, which corresponds to the total system, is possible only when the second emitted particle is frozen out from the fireball. This means that we adopt concept of the two-particle amplitude factorization. All these considerations complicate when we start with the two-particle Hamiltonian that contains terms where the coordinates are entagled, for instance the two-particle potential energy $V\left(\mathbf{x}_{a}-\mathbf{x}_{b}\right)$; but a discussion of this point is beyond the scope of the present letter (see [四 for details).

Owing to the particle-particle emission symmetry and to the commutation of the free evolution operators, we can write the measured two-particle momentum amplitude as

$$
\begin{aligned}
A_{\gamma_{a}, \gamma_{b}}\left(\mathbf{p}_{a}, \mathbf{p}_{b}, t_{a}, t_{b}\right)=\lim _{t \rightarrow \infty} & \frac{1}{\sqrt{2}} \int d^{3} x_{a} d^{3} x_{b} \phi_{\mathbf{p}_{a}, \mathbf{p}_{b}}^{\text {out }, *}\left(\mathbf{x}_{a}, \mathbf{x}_{b}, t\right) \\
\times & {\left[e^{-i\left[\hat{h}\left(\mathbf{x}_{a}\right)\left(t-t_{a}\right)+\hat{h}\left(\mathbf{x}_{b}\right)\left(t-t_{b}\right)\right]} \psi_{\gamma_{a}}\left(\mathbf{x}_{a}, t_{a}\right) \psi_{\gamma_{b}}\left(\mathbf{x}_{b}, t_{b}\right)+\right.} \\
& \left.+e^{i \alpha} e^{-i\left[\hat{h}\left(\mathbf{x}_{b}\right)\left(t-t_{a}\right)+\hat{h}\left(\mathbf{x}_{a}\right)\left(t-t_{b}\right)\right]} \psi_{\gamma_{a}}\left(\mathbf{x}_{b}, t_{a}\right) \psi_{\gamma_{b}}\left(\mathbf{x}_{a}, t_{b}\right)\right] .
\end{aligned}
$$

Now, we first relabel the variables of integration in the second term on the r.h.s. of this equation, what results in symmetrization (antisymmetrization) of the out-state. Secondly, we invert the evolution operator, which therefore acts on the out-state and brings it from asymptotic time $t$ to initial times $t_{a}$ and $t_{b}$. As a result, we come to the final expression of the two-particle amplitude:

$$
A_{\gamma_{a}, \gamma_{b}}\left(\mathbf{p}_{a}, \mathbf{p}_{b}, x_{a}^{0}, x_{b}^{0}\right)=\frac{1}{\sqrt{2}} \int d^{3} x_{a} d^{3} x_{b}\left[e^{i\left(p_{a} \cdot x_{a}+p_{b} \cdot x_{b}\right)}+e^{-i \alpha} e^{i\left(p_{a} \cdot x_{b}+p_{b} \cdot x_{a}\right)}\right] \psi_{\gamma_{a}}\left(x_{a}\right) \psi_{\gamma_{b}}\left(x_{b}\right),
$$

where $x_{a}^{0}=t_{a}$ and $x_{b}^{0}=t_{b}$. We therefore represent the measured two-particle momentum amplitude as a projection of a non-symmetrized two-particle wave function taken at emission times onto symmetrized (antisymmetrized) plane waves taken at emission times as well. 
The two-particle probability is obtained by averaging (52) and its complex conjugate with the density matrix defining the source. This density matrix is characterized by a probability distribution for the two-particle quantum numbers $\left(\gamma_{a}, \gamma_{b}\right)$ and by a distribution of emission times $\left(t_{a}, t_{b}\right)$. We write:

$$
\begin{aligned}
P_{2}\left(\mathbf{p}_{a}, \mathbf{p}_{b}\right) & =\overline{\left\langle\left|A_{\gamma_{a}, \gamma_{b}}\left(\mathbf{p}_{a}, \mathbf{p}_{b} ; t_{a}, t_{b}\right)\right|^{2}\right\rangle_{\gamma_{a}, \gamma_{b}}}= \\
& =\sum_{\gamma_{a} \gamma_{b}, \gamma_{a^{\prime}} \gamma_{b^{\prime}}} \int d t_{a} d t_{b} d t_{a^{\prime}} d t_{b^{\prime}} \rho_{\gamma_{a} \gamma_{a^{\prime}}} \rho_{\gamma_{b} \gamma_{b^{\prime}}} A_{\gamma_{a^{\prime}} \gamma_{b^{\prime}}}\left(\mathbf{p}_{a}, \mathbf{p}_{b} ; t_{a^{\prime}}, t_{b^{\prime}}\right) A_{\gamma_{a} \gamma_{b}}^{*}\left(\mathbf{p}_{a}, \mathbf{p}_{b} ; t_{a}, t_{b}\right)
\end{aligned}
$$

where anglular brackets on the r.h.s. of this equation mean averaging over the quantum numbers $\gamma_{a}, \gamma_{b}$ and the overline means averaging over the initial (emitting) times. We made the ansatz $\rho_{\gamma_{a} \gamma_{b}, \gamma_{a^{\prime}} \gamma_{b^{\prime}}}=\rho_{\gamma_{a} \gamma_{a^{\prime}}} \rho_{\gamma_{b} \gamma_{b^{\prime}}}$, which factorizes the initial density matrix $\rho_{\gamma_{a} \gamma_{b}, \gamma_{a^{\prime}} \gamma_{b^{\prime}}}$ in such a way that independent emission of the two particles is ensured.

According to (52), the probability consists of four terms, which we write as

$$
P_{2}\left(\mathbf{p}_{a}, \mathbf{p}_{b}\right)=P_{11}+P_{22}+P_{12}+P_{21}
$$

which have the structure $\left(a_{1}+a_{2}\right)\left(a_{1}+a_{2}\right)^{*}=a_{1} a_{1}^{*}+a_{2} a_{2}^{*}+a_{1} a_{2}^{*}+a_{2} a_{1}^{*}$. First diagonal term reads

$$
\begin{aligned}
P_{11}\left(p_{a}, p_{b}\right)= & \frac{1}{2} \int d^{4} x_{a} d^{4} y_{a} e^{i\left(p_{a} \cdot x_{a}-p_{a} \cdot y_{a}\right)}\left\langle\psi_{\gamma_{a}}\left(x_{a}\right) \psi_{\gamma_{a}}^{*}\left(y_{a}\right)\right\rangle_{\gamma_{a}} \\
& \times \int d^{4} x_{b} d^{4} y_{b} e^{i\left(p_{b} \cdot x_{b}-p_{b} \cdot y_{b}\right)}\left\langle\psi_{\gamma_{b}}\left(x_{b}\right) \psi_{\gamma_{b}}^{*}\left(y_{b}\right)\right\rangle_{\gamma_{b}} .
\end{aligned}
$$

We introduce the new momentum variables $K$ and $p$ as

$$
K=\frac{1}{2}\left(p_{a}+p_{b}\right), \quad p=p_{a}-p_{b} \Rightarrow p_{a}=K+\frac{p}{2}, \quad p_{b}=K-\frac{p}{2} .
$$

Using these variables and the space-time variables (40), $P_{11}$ can be rewritten in the form

$$
\begin{aligned}
P_{11}(p, K)= & \frac{1}{2} \int d^{4} X \int d^{4} x e^{\imath\left(K+\frac{1}{2} p\right) \cdot x}\left\langle\psi_{\gamma_{a}}\left(X+\frac{x}{2}\right) \psi_{\gamma_{a}}^{*}\left(X-\frac{x}{2}\right)\right\rangle_{\gamma_{a}} \\
& \times \int d^{4} Y \int d^{4} y e^{\imath\left(K-\frac{1}{2} p\right) \cdot y}\left\langle\psi_{\gamma_{b}}\left(Y+\frac{y}{2}\right) \psi_{\gamma_{b}}^{*}\left(Y-\frac{y}{2}\right)\right\rangle_{\gamma_{b}} .
\end{aligned}
$$

In the integrals over the variables $x$ and $y$, we immediately recognize the freeze-out Wigner density (41). But before rewriting this term let us note that $P_{22}$ can be obtained from $P_{11}$ by a mutual change of momenta $p_{a} \rightleftharpoons p_{b}$, which in turn results in changing $p$ to $-p$. Hence, to obtain $P_{22}$ from $P_{11}$ we need only to change the sign before $p$. Performing such a change, we come to an equality of the diagonal terms $P_{11}(p, K)=P_{22}(p, K)$. Putting together these two terms, we obtain

$$
P_{11}(p, K)+P_{22}(p, K)=\int d^{4} X S\left(X, K+\frac{p}{2}\right) \int d^{4} Y S\left(Y, K-\frac{p}{2}\right)
$$


i.e. the product of two single-particle probabilities (see Eq. (42) to registrate independently two particles with asymptotic momenta $\mathbf{p}_{a}=\mathbf{K}+\mathbf{p} / 2$ and $\mathbf{p}_{b}=\mathbf{K}-\mathbf{p} / 2$, respectively.

We now turn to considering of the cross term $P_{12}=a_{1} a_{2}^{*}$, which is a complex conjugate to the second cross-contribution $P_{21}=a_{1}^{*} a_{2}$, hence their sum is real. We have

$$
\begin{aligned}
P_{12}\left(p_{a}, p_{b}\right)= & \frac{1}{2} e^{i \alpha} \int d^{4} x_{a} d^{4} y_{a} e^{i\left(p_{a} \cdot x_{a}-p_{b} \cdot y_{a}\right)}\left\langle\psi_{\gamma_{a}}\left(x_{a}\right) \psi_{\gamma_{a}}^{*}\left(y_{a}\right)\right\rangle_{\gamma_{a}} \\
& \times \int d^{4} x_{b} d^{4} y_{b} e^{i\left(p_{b} \cdot x_{b}-p_{a} \cdot y_{b}\right)}\left\langle\psi_{\gamma_{b}}\left(x_{b}\right) \psi_{\gamma_{b}}^{*}\left(y_{b}\right)\right\rangle_{\gamma_{b}} .
\end{aligned}
$$

By the same change of variables as for diagonal terms, the exponents in the integrand can be rewritten as: $\left(p_{a} \cdot x_{a}-p_{b} \cdot y_{a}\right)=(p \cdot X+K \cdot x)$ and $\left(p_{b} \cdot x_{b}-p_{a} \cdot y_{b}\right)=(-p \cdot Y+K \cdot y)$. Using the definition of the source function (41), we obtain:

$$
P_{12}(p, K)=\frac{1}{2} e^{i \alpha} \int d^{4} X e^{i p \cdot X} S(X, K) \int d^{4} Y e^{-i p \cdot Y} S(Y, K) .
$$

The second cross term $P_{21}$, which is complex conjugate to $P_{12}$, is proportional to the exponent $\exp (-i \alpha)$, but the remaining factor is real. Hence, the sum of two cross terms looks like $P_{12}+P_{21}=\frac{1}{2} A e^{i \alpha}+\frac{1}{2} A e^{-i \alpha}=A \cos \alpha$, where $A$ is real. We are now ready to write the total expression for the two-particle probability

$$
\begin{aligned}
P_{2}(p, K) & =\int d^{4} X S\left(X, K+\frac{p}{2}\right) \int d^{4} Y S\left(Y, K-\frac{p}{2}\right) \\
& +\quad \cos \alpha \int d^{4} X e^{i p \cdot X} S(X, K) \int d^{4} Y e^{-i p \cdot Y} S(Y, K),
\end{aligned}
$$

and we finally get the two-particle correlator as

$$
C(p, K)=1+\cos \alpha \frac{\left|\int d^{4} X e^{i p \cdot X} S(X, K)\right|^{2}}{\int d^{4} X S\left(X, K+\frac{p}{2}\right) \int d^{4} Y S\left(Y, K-\frac{p}{2}\right)},
$$

where the source function $S(X, K)$ is defined in accordance with Eq. (41) and all integrations are taken at emission times or on the freeze-out hyper-surface.

\section{References}

[1] M. Gyulassy, S.K. Kauffmann and L.W. Wilson, Phys. Rev. C 20, 2267 (1979).

[2] D.H. Boal, C.-K. Gelbke and B.K. Jennings, Rev. Mod. Phys. 62, 553 (1990).

[3] U. Heinz and B.V. Jacak, Two-particle correlations in relativistic heavy-ion collisions, nucl-th/9902020.

[4] D. Anchishkin, U. Heinz and P. Renk, Phys. Rev. C 57, 1428 (1998), nucl-th/9710051. 
[5] H. Heiselberg, Phys. Lett. B 379, 27 (1996).

[6] U.A. Wiedemann and U. Heinz, nucl-th/9611031.

[7] S. Iwao, Progr. Theor. Phys. 83, 363 (1990).

D. Bonatsos, S.B. Drenska, P.P. Raychev, R.P. Roussev and Yu.F. Smirnov, J. Phys. G 17, L67 (1991);

[8] D.D. Coon, S. Yu and M. Baker, Phys. Rev. D 5, 1429 (1972).

M. Arik and D.D. Coon, J. Math. Phys. 17, 524 (1976).

[9] M. Chaichian, J.F. Gomez and P. Kulish, Phys. Lett. B 311, 93 (1993).

[10] L. Jenkovszky, M. Kibler and A. Mishchenko, Mod. Phys. Lett. A 10, 51 (1995), hep-th/9407071.

[11] A.M. Gavrilik, J. Phys. A 27, L91 (1994); A.M. Gavrilik, in: "Non-Euclidean Geometry in Modern Physics" (L. Jenkovszky, ed.), Kiev, 1997, pp. 183-192, hep-ph/9712411.

[12] A.M. Gavrilik and N.Z. Iorgov, Ukrain. J. Phys. 43, 1526 (1998), hep-ph/9807559.

[13] O.W. Greenberg, D.M. Greenberger and T.V. Greenbergest, in Quantum Coherence and Reality, eds. J.S. Anandan and J.L. Safco (World Scientific, Singapore, 1994).

[14] S.S. Avancini and G. Krein, J. Phys. A 28, 685 (1995).

[15] M. Arik and D.D. Coon, as quoted in [8].

V. Kuryshkin, Ann. Found. Louis de Broglie 5, 111 (1980)

P. Kulish and E. Damaskinsky, J. Phys. A 23, L415 (1990).

[16] O.W. Greenberg, Phys. Rev. Lett. 64, 705 (1990); Phys. Rev. D 43, 4111 (1991).

R.N. Mohapatra, Phys. Lett. B 242, 407 (1990).

[17] S. Chaturvedi, A.K. Kapoor, R. Sandhya, V. Srinivasan and R. Simon, Phys. Rev. A 43, 4555 (1991).

[18] A.J. Macfarlane, J. Phys. A 22, 4581 (1989).

L. Biedenharn, J. Phys. A 22, L873 (1989).

[19] M. Arik, Z. Phys. C 51, 627 (1991).

D. Fairlie and C. Zachos, Phys. Lett. B 256, 43 (1991).

S. Meljanac and A. Perica, Mod. Phys. Lett. A 9, 3293 (1994).

[20] V.I. Man'ko, G. Marmo, S. Solimeno and F. Zaccaria, Phys. Lett. A 176, 173 (1993).

J.W. Goodison and D.J. Toms, Phys. Lett. A 195, 38 (1994).

[21] T. Altherr and T. Grandou, Nucl. Phys. B 402, 195 (1993).

[22] M. Salerno, Phys. Rev. E 50, 6 (1994). 